\title{
On multiplicative magic squares
}

\author{
Javier Cilleruelo* \\ Instituto de Ciencias Matemáticas (CSIC-UAM-UC3M-UCM) and \\ Departamento de Matemáticas \\ Universidad Autónoma de Madrid \\ 28049 Madrid, Spain \\ franciscojavier.cilleruelo@uam.es \\ Florian Luca ${ }^{\dagger}$ \\ Instituto de Matemáticas \\ Universidad Nacional Autónoma de México \\ C.P. 58089 Morelia, Michoacan, México \\ fluca@matmor.unam.mx
}

Submitted: Apr 22, 2008; Accepted: Jan 8, 2010; Published: Jan 14, 2010

Mathematics Subject Classifications: 11N99,05B15

\begin{abstract}
In this note, we give a lower bound for the distance between the maximal and minimal element in a multiplicative magic square of dimension $r$ whose entries are distinct positive integers.
\end{abstract}

\section{Introduction}

Let $A=[A(i, j)]_{1 \leqslant i, j \leqslant r}$ be a square matrix with positive integer entries. We say that $A$ is an additive magic square of order $r$ if the sums of the entries in each row, column, and the two diagonals are all equal. We write $s(A)$ for this common value.

A multiplicative magic square has the property that the products of the entries in each row, column, and the two diagonals are all equal. We write $p(A)$ for this common value. We shall deal only with magic squares whose entries are distinct positive integers.

The most popular additive magic squares of order $r$ are those whose entries are the first $r^{2}$ positive integers. Clearly, there are no multiplicative magic squares with this

* J. C. was supported in part by Project MTM2008-03880 from MINCYT (Spain) and the joint Madrid Region-UAM project TENU3 (CCG08-UAM/ESP-3906).

${ }^{\dagger}$ F. L. was supported in part by Grants SEP-CONACyT 46755 and PAPIIT IN 100508. 
property and indeed it is not difficult to guess that the entries in a multiplicative magic square cannot be very close. In this note, we take a closer look at this problem.

The motivation of this note is to understand better how close the divisors of a positive integer can be. It has been proved in [2] and [3] that an interval of length $n^{\frac{1}{4}-\frac{1}{8[k / 2]+4}}$ contains at most $k$ divisors of $n$ larger than $\sqrt{n}$. It is known that the exponent above is sharp for $k=1,2,3$ but not for greater values of $k$. A multidimensional version of this problem is to find a lower bound for the distance between the vectors $\left(a_{11}, \ldots a_{m 1}\right), \ldots,\left(a_{1 k}, \ldots, a_{m k}\right)$ satisfying $a_{1 i} \cdots a_{m i}=n, i=1, \ldots, k$. The problem that we study in this note is therefore just a toy version of the above more general problem.

Let $X=\left[x_{i j}\right]_{1 \leqslant i, j \leqslant r}$ be a multiplicative magic square whose entries are distinct positive integers, and let $x_{M}$ and $x_{m}$ be the largest and respectively smallest entry in $X$. We prove some nontrivial lower bound for $x_{M}-x_{m}$. For $r=3$, we get a very precise result.

Theorem 1. In a multiplicative magic square $X$ of order 3 with distinct entries we have that

$$
x_{M}-x_{m} \geqslant x_{m}^{3 / 4} \text {. }
$$

Furthermore, there exists an infinite family $(X(n))_{n \geqslant 1}$ of multiplicative magic squares of order 3 with distinct entries such that

$$
x_{M}(n)-x_{m}(n) \leqslant x_{m}^{3 / 4}(n)(1+o(1)) \quad \text { as } n \rightarrow \infty .
$$

For $r=4$, we obtain the true minimal order of magnitude for the above difference.

Theorem 2. In an multiplicative magic square $X$ of order 4 with distinct entries we have that

$$
x_{M}-x_{m} \geqslant 5^{5 / 12} x_{m}^{1 / 2} .
$$

Furthermore, there exists an infinite family of multiplicative magic squares $(X(n))_{n \geqslant 1}$ of order 4 with distinct entries such that

$$
x_{M}(n)-x_{m}(n) \leqslant 6 x_{m}^{1 / 2}(n)(1+o(1)) \quad \text { as } n \rightarrow \infty .
$$

An example of such a family is

$$
X(n)=\left[\begin{array}{cccc}
(n+2)(n+4) & (n+3)(n+7) & (n+1)(n+6) & n(n+5) \\
(n+1)(n+5) & n(n+6) & (n+2)(n+7) & (n+3)(n+4) \\
n(n+7) & (n+1)(n+4) & (n+3)(n+5) & (n+2)(n+6) \\
(n+3)(n+6) & (n+2)(n+5) & n(n+4) & (n+1)(n+7)
\end{array}\right]
$$

A more general family generated by the ortogonal latin square with rows $[20,33,12,01]$, $[11,02,23,30],[03,10,31,22]$ and $[32,21,00,13]$ is given by

$$
X(n, m)=\left[\begin{array}{llll}
(n+2)(m+0) & (n+3)(m+3) & (n+1)(m+2) & (n+0)(m+1) \\
(n+1)(m+1) & (n+0)(m+2) & (n+2)(m+3) & (n+3)(m+0) \\
(n+0)(m+3) & (n+1)(m+0) & (n+3)(m+1) & (n+2)(m+2) \\
(n+3)(m+2) & (n+2)(m+1) & (n+0)(m+0) & (n+1)(m+3)
\end{array}\right] .
$$


It should be noted that these multiplicative magic squares are almost additive magic squares since the sums of the entries in each row, column, and diagonal differ by at most 6. We don't know if 6 is the smallest possible such value, but it is not difficult to see that a magic square of order 4 with distinct entries cannot be simultaneously multiplicative and additive. To see this, observe that if $X$ is a additive magic square, then

$$
\begin{aligned}
2\left(x_{11}+x_{44}-x_{32}+x_{23}\right)= & \left(x_{11}+x_{12}+x_{13}+x_{14}\right)+\left(x_{41}+x_{42}+x_{43}+x_{44}\right) \\
& -\left(x_{12}+x_{22}+x_{32}+x_{42}\right)-\left(x_{13}+x_{23}+x_{33}+x_{43}\right) \\
& +\left(x_{11}+x_{22}+x_{33}+x_{44}\right)-\left(x_{14}+x_{23}+x_{32}+x_{41}\right) \\
= & 0 .
\end{aligned}
$$

So, $x_{11}+x_{44}=x_{32}+x_{23}$. If in addition $X$ is a multiplicative magic square we have, for similar reasons, that $x_{11} x_{44}=x_{32} x_{23}$, so $\left\{x_{11}, x_{44}\right\}=\left\{x_{23}, x_{32}\right\}$, which is impossible since these four entries must be distinct.

The method we use to obtain the lower bounds in the Theorems 2 and 3 turns out to be too complicated for $r \geqslant 5$. Thus, when $r \geqslant 5$, we apply a different method which leads to a weaker result.

Theorem 3. In a multiplicative magic square $X$ of order $r \geqslant 5$ with distinct entries we have

$$
x_{M}-x_{m} \geqslant C_{r} x_{m}^{1 /(r-1)}
$$

for some positive constant $C_{r}$.

Of course the exponent $1 /(r-1)$ is theorem above is not sharp, at least for $r=3$ and $r=4$. This motives the first question that we leave for the reader:

Problem 1. What is the best exponent $e_{r}$ in Theorem 3?

Our results Theorem 1 and Theorem 2 show that $e_{3}=3 / 4$ and $e_{4}=1 / 2$.

Problem 2. Are there additive-multiplicative magic squares of order $r=5$ with distinct entries?

We have seen that the answer is negative for $r=4$. On the contrary, Horner [4] found an additive and multiplicative magic square of order $r=8$ with distinct entries.

One of the topics of interest concerned with additive magic squares is to enumerate them.

Problem 3. Find an asymptotic estimate for the number of multiplicative magic squares $A$ of order $r$ (for example when $r=3$ ) having $p(A) \leqslant x$.

Acknowledgement. We thank the anonymous referees for comments which improved the quality of this paper. 


\section{Proofs}

The multiplicative magic squares can be described in terms of the additive ones in the following way:

We write $n^{A}$ for the multiplicative magic square given by

$$
n^{A}=\left[n^{A(i, j)}\right]_{1 \leqslant i, j \leqslant r} .
$$

If we write $\times$ for the entrywise multiplication of the magic squares, then we have the following properties:

(i) $n^{A} \times n^{B}=n^{A+B}$;

(ii) $n^{A} \times m^{A}=(n m)^{A}$.

Each multiplicative magic square can be factored uniquely as $\prod_{s=1}^{t} p_{s}{ }^{A_{p_{s}}}$, where $p_{1}<$ $\cdots<p_{t}$ are primes and the $A_{p_{s}}{ }^{\prime}$ s are additive magic squares for $s=1, \ldots, t$.

The additive magic squares of nonnegative integers form the set of integral points inside a pointed polyhedral cone (see [5]). Thus, the additive magic squares of order $r$ have a minimal base of irreducible magic squares called a Hilbert base $H_{r}=\left\{B_{l}: l \in L\right\}$ in such a way that every additive magic square $A$ with nonnegative integer entries can be written as

$$
A=\sum_{l \in L} c_{l} B_{l}, \quad \text { for some nonnegative } c_{l} \in \mathbb{Z} .
$$

The Hilbert bases for the magic squares of orders 3 and 4 have been calculated in [1].

The basis $H_{3}$ consists of the following magic squares:

$$
B_{1}=\left[\begin{array}{lll}
2 & 0 & 1 \\
0 & 1 & 2 \\
1 & 2 & 0
\end{array}\right], B_{2}=\left[\begin{array}{lll}
0 & 2 & 1 \\
2 & 1 & 0 \\
1 & 0 & 2
\end{array}\right], B_{3}=\left[\begin{array}{lll}
1 & 0 & 2 \\
2 & 1 & 0 \\
0 & 2 & 1
\end{array}\right], B_{4}=\left[\begin{array}{lll}
1 & 2 & 0 \\
0 & 1 & 2 \\
2 & 0 & 1
\end{array}\right], B_{5}=\left[\begin{array}{lll}
1 & 1 & 1 \\
1 & 1 & 1 \\
1 & 1 & 1
\end{array}\right] .
$$

The basis $H_{4}$ consists of the following magic squares

$$
\begin{aligned}
& B_{1}=\left[\begin{array}{llll}
1 & 0 & 0 & 0 \\
0 & 0 & 0 & 1 \\
0 & 1 & 0 & 0 \\
0 & 0 & 1 & 0
\end{array}\right] \quad B_{2}=\left[\begin{array}{llll}
1 & 0 & 0 & 0 \\
0 & 0 & 1 & 0 \\
0 & 0 & 0 & 1 \\
0 & 1 & 0 & 0
\end{array}\right] \quad B_{3}=\left[\begin{array}{llll}
0 & 0 & 1 & 0 \\
1 & 0 & 0 & 0 \\
0 & 1 & 0 & 0 \\
0 & 0 & 0 & 1
\end{array}\right] \quad B_{4}=\left[\begin{array}{llll}
0 & 0 & 0 & 1 \\
1 & 0 & 0 & 0 \\
0 & 0 & 1 & 0 \\
0 & 1 & 0 & 0
\end{array}\right] \quad B_{5}=\left[\begin{array}{llll}
0 & 1 & 0 & 0 \\
0 & 0 & 1 & 0 \\
1 & 0 & 0 & 0 \\
0 & 0 & 0 & 1
\end{array}\right]
\end{aligned}
$$

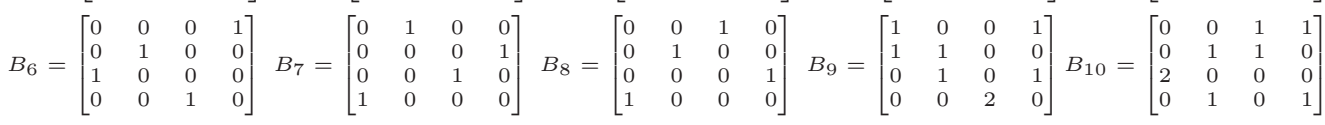

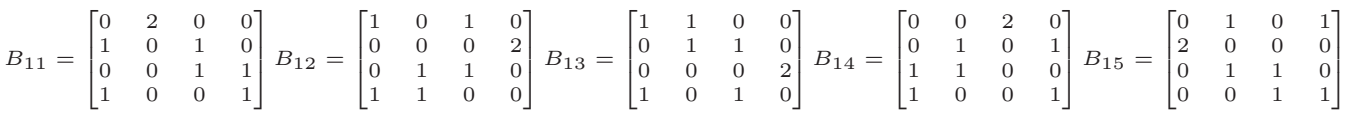

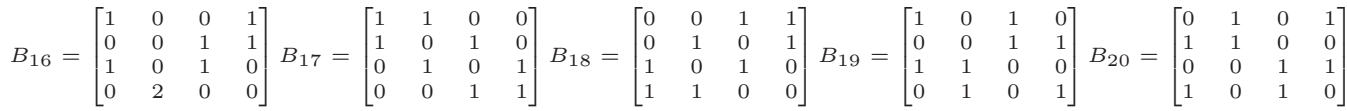

We recall that for us $X=\left[x_{i j}\right]_{1 \leqslant i, j, \leqslant r}$ is a multiplicative magic square whose entries are distinct positive integers and that $x_{M}$ and $x_{m}$ denote the largest and smallest entry in $X$, respectively. We start with the following preliminary result. 
Lemma 1. Let $R=\left\{\left(i j, i^{\prime} j^{\prime}\right)\right\}$ be a collection of pairs of positions in a magic square of order $r$ having the following property:

$$
\sum_{\left(i j, i^{\prime} j^{\prime}\right) \in R} \min \left\{B_{l}(i, j), B_{l}\left(i^{\prime}, j^{\prime}\right)\right\} \geqslant k s\left(B_{l}\right) \quad \text { for all } B_{l} \in H_{r} .
$$

Let $X=\left[x_{i j}\right]_{1 \leqslant i, j \leqslant r}$ be a multiplicative magic square of order $r$ with distinct entries. Then the inequality

$$
x_{M}-x_{m} \geqslant x_{m}^{k r /|R|}
$$

holds.

Proof. Write $X=\prod_{s=1}^{t} p_{s}^{A_{p_{s}}}=\prod_{s=1}^{t} p_{s}^{\sum_{l} c_{l, p_{s}} B_{l}}$, where $p_{1}<\cdots<p_{t}$ are distinct primes and $A_{p_{s}}$ 's are additive magic squares for $s=1, \ldots, t$. Thus, $x_{i j}=\prod_{s=1}^{t} p^{\sum_{l} c_{l, p_{s}} B_{l}(i, j)}$. Then, since $x_{i j} \neq x_{i^{\prime} j^{\prime}}$,

$$
\begin{aligned}
& \left|x_{i j}-x_{i^{\prime} j^{\prime}}\right|=\left|\prod_{s=1}^{t} p_{s} \sum_{l} c_{l, p_{s}} B_{l}(i, j)-\prod_{s=1}^{t} p_{s} \sum_{l} c_{l, p_{s}} B_{l}\left(i^{\prime}, j^{\prime}\right)\right| \\
& \geqslant \prod_{s=1}^{t} p_{s} \min \left\{\sum_{l} c_{l, p_{s}} B_{l}(i, j), \sum_{l} c_{l, p_{s}} B_{l}\left(i^{\prime}, j^{\prime}\right)\right\} \\
& \geqslant \prod_{s=1}^{t} p_{s}^{\sum_{l} c_{l, p_{s}} \min \left\{B_{l}(i, j), B_{l}\left(i^{\prime}, j^{\prime}\right)\right\}}
\end{aligned}
$$

Thus,

$$
\begin{aligned}
\left(x_{M}-x_{m}\right)^{|R|} & \geqslant \prod_{\left(i j, i^{\prime} j^{\prime}\right) \in R}\left|x_{i j}-x_{i^{\prime} j^{\prime}}\right| \\
& \geqslant \prod_{s=1}^{t} p_{s} \sum_{l} c_{l, p_{s}} \sum_{\left(i j, i^{\prime} j^{\prime}\right) \in R} \min \left\{B_{l}(i, j), B_{l}\left(i^{\prime}, j^{\prime}\right)\right\} \\
& \geqslant \prod_{s=1}^{t} p_{s} \sum_{l} c_{l, p_{s}} k s\left(B_{l}\right)=\prod_{s=1}^{t} p_{s}^{s\left(A_{p_{s}}\right) k} .
\end{aligned}
$$

We finish the proof by noting that

$$
x_{m}^{r} \leqslant \prod_{i=1}^{r} x_{1 i}=\prod_{s=1}^{t} p_{s}^{\sum_{i=1}^{r} \alpha_{p_{s}}(1, i)}=\prod_{s=1}^{t} p_{s}{ }^{s\left(A_{p_{s}}\right)} .
$$

Proof of Theorem 1. We take $R=\{(11,22),(13,22),(31,22),(33,22)\}$ in Lemma 1 for $r=3$. Observe that $k=1$.

The family given by

$$
X(n)=n^{B_{1}} \times(n+1)^{B_{2}} \times(n+2)^{B_{3}} \times(n+3)^{B_{4}}
$$

for all $n \geqslant 1$ satisfies the second part of the theorem. 
Proof of Theorem 2. We now take $R=\cup_{m=1}^{8} R_{m}$, where for each $m=1, \ldots, 8$, the set $R_{m}$ consists of all the 6 subsets of pairs of positions $\left(i j, i^{\prime} j^{\prime}\right)$ such that $B_{m}(i, j)=B_{m}\left(i^{\prime}, j^{\prime}\right)=1$. Let us observe that in the notations of Lemma 1 , we have $k=6$. Lemma 1 now gives us the inequality

$$
x_{M}-x_{m} \geqslant x_{m}^{1 / 2} .
$$

To improve a bit on this inequality (on the multiplicative constant, not on the exponent $1 / 2$ ), observe that we can write

$$
\begin{aligned}
\prod_{\left(i j, i^{\prime} j^{\prime}\right) \in R}\left|x_{i j}-x_{i^{\prime} j^{\prime}}\right| & =\prod_{m=1}^{8} \prod_{\left(i j, i^{\prime} j^{\prime}\right) \in R_{m}}\left|x_{i j}-x_{i^{\prime} j^{\prime}}\right| \leqslant\left(\frac{1}{25 \sqrt{5}}\left(x_{M}-x_{m}\right)^{6}\right)^{8} \\
& =\frac{1}{5^{20}}\left(x_{M}-x_{m}\right)^{48}
\end{aligned}
$$

In the above chain of inequalities, we have used the easy exercise (left to the reader) that if $0 \leqslant \alpha_{1} \leqslant \alpha_{2} \leqslant \alpha_{3} \leqslant \alpha_{4} \leqslant 1$, then $\prod_{i<j}\left|\alpha_{i}-\alpha_{j}\right| \leqslant 1 /(25 \sqrt{5})$.

The family

$X(n)=n^{B_{6}} \times(n+1)^{B_{3}} \times(n+2)^{B_{2}} \times(n+3)^{B_{7}} \times(n+4)^{B_{1}} \times(n+5)^{B_{4}} \times(n+6)^{B_{8}} \times(n+7)^{B_{5}}$

for all positive integers $n$ satisfies the second part of the theorem and corresponds to the family (1) described in the introduction.

Proof of Theorem 3. We proceed by contradiction. We let $s$ be the smallest element in the magic square and assume that $s$ is on row $i$ and column $j$. Write $x_{k l}=s+s_{k l}$ for all $k, l \in\{1, \ldots, r\}$ and expand the products on row $i$ and column $j$ as follows:

$$
\begin{aligned}
\prod_{l=1}^{r} x_{i l} & =\prod_{l=1}^{r}\left(s+s_{i l}\right) \\
& =s\left(s^{r-1}+s^{r-2} \sum_{\substack{1 \leqslant l \leqslant r \\
l \neq j}} s_{i l}+s^{r-3} \sum_{\substack{1 \leqslant l_{1}<l_{2} \leqslant r \\
l_{1} \neq j \neq l_{2}}} s_{i l_{1}} s_{i l_{2}}+\cdots+\prod_{\substack{1 \leqslant l \leqslant r \\
l \neq j}} s_{i l}\right)
\end{aligned}
$$

and similarly for column $j$. Since the two products obtained in this way are equal and since $s$ is a common factor of both of them, we get that

$$
\begin{array}{r}
\left|\sum_{\substack{1 \leqslant l \leqslant r \\
l \neq j}} s_{i l}-\sum_{\substack{1 \leqslant l \leqslant r \\
l \neq i}} s_{l j}\right|<s^{-1}\left|\sum_{\substack{1 \leqslant l_{1}<l_{2} \leqslant r \\
l_{1} \neq j \neq l_{2}}} s_{i l_{1}} s_{i l_{2}}-\sum_{\substack{1 \leqslant l_{1}<l_{2} \leqslant r \\
l_{1} \neq j \neq l_{2}}} s_{l_{1} j} s_{l_{2} j}\right| \\
+\cdots+s^{-(r-2)}\left|\prod_{\substack{1 \leqslant l \leqslant r \\
l \neq j}} s_{i l}-\prod_{\substack{1 \leqslant l \leqslant r \\
l \neq i}} s_{l j}\right| .
\end{array}
$$


We now assume that $0<s_{k l}<2^{-(r-1) / 2} s^{1 /(r-1)}$ holds for all $k, l \in\{1, \ldots, r\}$ except for $(k, l)=(i, j)$ in order to get a contradiction. We then get that the right hand side above is

$$
<2^{-(r-1)} s^{-1+2 /(r-1)}\left(\left(\begin{array}{c}
r-1 \\
2
\end{array}\right)+\left(\begin{array}{c}
r-1 \\
3
\end{array}\right)+\cdots+\left(\begin{array}{c}
r-1 \\
r-1
\end{array}\right)\right)<1
$$

therefore

$$
\sum_{l=1}^{r} s_{i l}=\sum_{l=1}^{r} s_{l j} .
$$

We now proceed by induction on $t$ to show that the two th symmetric polynomials

$$
\sum_{1 \leqslant l_{1}<\cdots<l_{t} \leqslant r} s_{i l_{1}} \cdots s_{i l_{t}}=\sum_{1 \leqslant l_{1}<\cdots<l_{t} \leqslant r} s_{l_{1} j} \cdots s_{l_{t} j}
$$

in the $\left(s_{i l}\right)_{1 \leqslant l \leqslant r}$ and $\left(s_{l j}\right)_{1 \leqslant l \leqslant r}$ are equal. Formula (3) shows that this holds when $t=1$ and by induction it is enough to show that the two th symmetric polynomials on the sets of $r-1$ dimensional indeterminates $\left(s_{i l}\right)_{\substack{1 \leqslant l \leqslant r \\ l \neq j}}$ and $\left(s_{l j}\right)_{\substack{1 \leqslant l \leqslant r \\ l \neq i}}$ are equal. Assuming that $t \geqslant 2$ and that the above equality holds for $t-1<r-1$, then equating again the two products shown at (2) for the $i$ th row with the analogous one obtained for the $j$ th row, we get

$$
\begin{aligned}
& \left|\sum_{\substack{1 \leqslant l_{1}<\ldots<l_{t} \leqslant r \\
l_{k} \neq j, 1 \leqslant k \leqslant t}} s_{i l_{1}} \cdots s_{i l_{t}}-\sum_{\substack{1 \leqslant l_{1}<\cdots<l_{t} \leqslant r \\
l_{k} \neq i, 1 \leqslant k \leqslant t}} s_{l_{1} j} \cdots s_{l_{t} j}\right| \\
& \leqslant s^{-1}\left|\sum_{\substack{1 \leqslant l_{1}<\ldots<l_{t+1} \leqslant r \\
l_{k} \neq j, 1 \leqslant k \leqslant t+1}} s_{i l_{1}} \cdots s_{i l_{t+1}}-\sum_{\substack{1 \leqslant l_{1}<\cdots<l_{t+1} \leqslant r \\
l_{k} \neq i, 1 \leqslant k \leqslant t+1}} s_{l_{1} j} \cdots s_{l_{t+1} j}\right| \\
& +\cdots+s^{-(r-1-t)}\left|\prod_{\substack{1 \leqslant l \leqslant r \\
l \neq j}} s_{i l}-\prod_{\substack{1 \leqslant l \leqslant r \\
l \neq i}} s_{l j}\right| .
\end{aligned}
$$

Using again the fact that $s \geqslant 1$ and $0<s_{k l}<2^{-(r-1) / 2} s^{1 /(r-1)}$ whenever $(k, l) \neq(i, j)$, we get that

$$
\begin{aligned}
& \left|\sum_{\substack{1 \leqslant l_{1}<\cdots<l_{t} \leqslant r \\
l_{k} \neq j, 1 \leqslant k \leqslant t}} s_{i l_{1}} \cdots s_{i l_{t}}-\sum_{\substack{1 \leqslant l_{1}<\cdots<l_{t} \leqslant r \\
l_{j} \neq i, 1 \leqslant k \leqslant t}} s_{l_{1} j} \cdots s_{l_{t} j}\right| \\
& <2^{-(r-1)} s^{-1+(t+1) /(r-1)}\left(\left(\begin{array}{c}
r-1 \\
t+1
\end{array}\right)+\cdots+\left(\begin{array}{c}
r-1 \\
r-1
\end{array}\right)\right)<1,
\end{aligned}
$$


therefore relation (4) holds for $t$ as well. Since this is true for all $t=1, \ldots, r$, we deduce that the two polynomials

$$
\prod_{l=1}^{r}\left(X-s_{i l}\right) \quad \text { and } \quad \prod_{l=1}^{r}\left(X-s_{l j}\right)
$$

are equal. In particular, the entries from row $i$ are a permutation of the entries from column $j$, but this is not allowed since the union of these entries should be a set of $2 r-1$ distinct integers. This completes the proof of Theorem 3.

\section{References}

[1] M. Ahmed, "Algebraic combinatorics of magic squares", Preprint posted at arXiv:math.CO/0405476.

[2] J. Cilleruelo and J. Jiménez, "The hyperbola $x y=N$ ", Journal of Théorie des Nombres of Bordeaux, vol 12, n: 1 (2000)

[3] J. Cilleruelo and G. Tenenbaum, "An overlapping theorem with applications", Publicacions Matematiques, Primeras Jornadas de Teoría de Números (2007)

[4] W. W. Horner, "Addition-Multiplication Magic Square of Order 8", Scripta Math. 21, 23-27, 1955.

[5] R.P. Stanley, Enumerative Combinatorics, Vol. I, Cambridge Univ. Press, Cambridge, 1997. 